\title{
NSCLC-Impfung als Erhaltungstherapie bleibt experimentell
}

\section{Immer noch sind die Rezidivraten nach der Erstlinientherapie des nichtkleinzelligen Lungenkarzinoms (NSCLC) hoch. Ist die Vakzinierung eine Option?}

D ie therapeutische Vakzine Belagenpumatucel-L ist eine allogene Tumorzell-Vakzine auf Basis von 4 verschiedenen NSCLC-Zelllinien. In einer Phase-III-Studie mit insgesamt $532 \mathrm{~Pa}$ tienten mit NSCLC im Stadium III/IV wurde die Wirkung der Vakzine untersucht. Die Patienten hatten eine platinbasierte Induktionstherapie erhalten und bislang keinen Progress entwickelt. Randomisiert wurde ihnen 1-4 Monate nach Ende der Induktionstherapie entweder eine Erhaltungstherapie mit bis zu 20 Zyklen Belagenpumatucel-L $(\mathrm{n}=270)$ oder Placebo $(n=262)$ intradermal injiziert.
Nach der zweiten Interimsanalyse wurde die Studie beendet, weil der erwartete Überlebensvorteil ausblieb. Der primäre Endpunkt medianes Gesamtüberleben lag bei 20,3 Monaten im Impfund 17,8 Monaten im Kontrollarm (Hazard Ratio [HR] 0,94; $\mathrm{p}=0$,594). Auch beim progressionsfreien Überleben unterschieden sich die Gruppen nicht (4,3 vs. 4,0 Monate; HR 0,99; $p=0,947)$. Das Sicherheitsprofil entsprach dem aus vorangegangenen Studien. Grad-3/4-Nebenwirkungen gingen meist nicht auf die Injektionen zurück. In einem Fall führten sie aber zum Abbruch der Impfungen.

Eine multivariate Analyse zeigte, dass die Zeit zwischen Ende der Induktionschemotherapie und Randomisierung das Überleben beeinflusste. Erfolgte die Randomisierung binnen 12 Wochen nach Ende der Chemotherapie, zeigte sich bei aktiver Impfung ein Überle- bensvorteil (median 20,7 vs. 13,4 Monate; HR 0,77; $p=0,002$ ). Auch eine vorangegangene Strahlentherapie war prognostisch günstig (28,4 vs. 16,0 Monate; HR 0,$61 ; \mathrm{p}=0,032$ ).

Fazit: Die Phase-III-Studie konnte nicht die hoffnungsvoll stimmenden Ergebnisse aus der vorangegangenen Phase-IIStudie bestätigen. Die präspezifizierten Subgruppenanalysen weisen aber darauf hin, dass Patienten mit einem Beginn der Tumorimpfung innerhalb von 12 Wochen nach Ende der Chemotherapie bzw. mit vorangegangener Strahlentherapie doch länger überleben. Entsprechend sollten weitere Studien durchgeführt werden.

Friederike Klein

Giaccone $\mathrm{G}$ et al. A phase III study of belagenpumatucel- $L$, an allogeneic tumour cell vaccine, as maintenance therapy for non-small cell lung cancer. Eur J Cancer. 2015;51(16):2321-9.

\section{NSCLC: Relativ gute Prognose trotz Stadium IV}

\section{Patienten mit nichtkleinzelligem Lungenkarzinom (NSCLC) im Stadi- um IV leben oft nicht mehr lange. Trotzdem sollte man genauer hin- sehen, ehe man die Prognose stellt.}

$\mathrm{m}$ Stadium IV eines NSCLC werden bezüglich der Prognose Subgruppen mit intrathorakalen (M1a) und mit Fernmetastasen (M1b) - mit einem medianen Gesamtüberleben von acht bzw. sechs Monaten - unterschieden. Innerhalb der M1b-Gruppe kann es jedoch erhebliche Unterschiede hinsichtlich Zahl und Art der befallenen Organe geben.

Basis der vorliegenden Analyse waren die Daten aller NSCLC-Patienten im Stadium IV mit histologisch gesichertem Befund aus den Jahren 2006-2012 im niederländischen Krebsregister $(\mathrm{n}=11.094)$. In den multivariaten Analysen wurde auch eine Subgruppe untersucht, bei der das Staging mittels Positronenemissionstomografie (PET) erfolgt war, denn diese könnte wegen ihrer Genauigkeit zum Upstaging führen.
Das mediane Gesamtüberleben der Patienten betrug bei einem befallenen Organ $(n=5.676) 6,7$, bei zwei befallenen Organen $(\mathrm{n}=3.280) 4,3$ und bei drei befallenen Organen $(\mathrm{n}=2.138) 2,8$ Monate $(\mathrm{p}<0,001)$. Die Hazard Ratio (HR) für den Befall von zwei gegenüber einem Organ lag bei $1,33(\mathrm{p}<0,001)$ und für den Befall von drei gegenüber einem Organ bei $1,91(\mathrm{p}<0,001)$. In der Kohorte mit PET-Staging $(\mathrm{n}=1.517)$ waren die Ergebnisse ähnlich: Die HR betrug 1,40 bzw. 2,17; das Gesamtüberleben für die einzelnen Gruppen lag bei 8,6, 5,7 bzw. 3,8 Monate $(\mathrm{p}<0,001)$.

War nur ein Organ von Metastasen befallen, betrug das Gesamtüberleben für einen niedrigen gegenüber einem hohen TN-Status 8,5 gegenüber 6,5 Monaten (HR 1,40; p < 0,001), für die Patienten mit PET-Staging 11,6 gegenüber 8,2 Monaten (HR 1,62; $\mathrm{p}<0,001)$.

Fazit: NSCLC-Patienten im Stadium IV haben eine bessere Prognose, wenn bei ihnen Metastasen nur ein Organ befal-

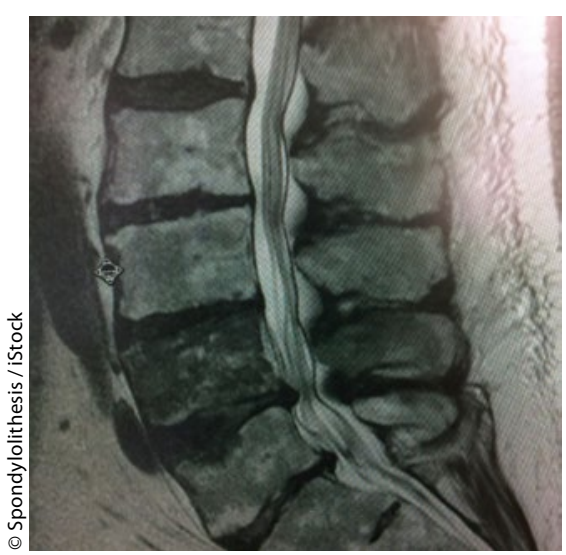

Am häufigsten waren zum Diagnosezeitpunkt Knochenmetastasen.

len. Das gilt besonders bei niedrigem TN-Status. Dabei scheint es unerheblich zu sein, ob das Staging mit oder ohne PET erfolgte. Diese Patienten mit unilokulärer Metastasierung sollten separat betrachtet werden.

Christina Berndt

Hendriks LE et al. Single organ metastatic disease and local disease status, prognostic factors for overall survival in stage IV non-small cell lung cancer: Results from a populationbased study. Eur J Cancer. 2015;51(17):2534-44. 